\title{
INTERACTIVE MARKER-BASED AUGMENTED REALITY FOR CPR TRAINING
}

\author{
Poonpong Boonbrahm ${ }^{1 *}$, Charlee Kaewrat ${ }^{1}$, Salin Boonbrahm ${ }^{1}$ \\ ${ }^{1}$ School of Informatics, Walailak University, Nakorn si Thammarat 80160, Thailand
}

(Received: November 2018 / Revised: January 2019 / Accepted: September 2019)

\begin{abstract}
CPR, or Cardiopulmonary Resuscitation, is a lifesaving technique useful for the case in which someone's heartbeat or breathing has stopped due to heart attack. Without proper CPR, nine out of ten patients die. The American Heart Association recommends CPR with chest compressions in the event of witnessing such an incident. For proper CPR training, taking a class with a CPR instructor is usually the best choice, but it is not practical and costly for mass training, especially in schools and universities. There are many new techniques available that can replace traditional CPR training and Augmented Reality (AR) is one of them. AR is the technology that integrates virtual objects or environments, created by digital technology, with the real world. Augmented Reality using marker-based technique is a good option, since a trainee can have a realistic look at the patient, know the position of the hand on the chest, identify the number of chest compressions per minute, and also know the pressure that he or she puts on the chest. Besides that, the status of the operation can be displayed along with a recording system for analysis. In this research, we chose marker-based AR due to its precision in distance measurement. For measuring the pressure on the chest, we use a marker-marker interaction technique. Unity 3D cross-platform game engine and Qualcomm's Vuforia-an augmented reality software development kit (SDK) for mobile devices that enables the creation of augmented reality applications - are required. The results from our experiment with a group of people with nonCPR training confirm that the configuration increases the speed and accuracy of CPR training.
\end{abstract}

Keywords: Augmented reality; CPR Training; Marker-based AR; Marker-Marker Interaction

\section{INTRODUCTION}

In the past few years, there have been some significant changes in the field of healthcare thanks to advanced information technology, both in terms of quality and variety of services. One of the techniques that can be applied to healthcare services, and that seems to be the most promising, is Augmented Reality (AR). AR is the technology that integrates virtual objects or environments, created by digital technology, with the real world. With AR technology, digital objects, such as 3D models, text, video, and sound generated by a digital computer can overlay on top of the real world at the exact point where it is designated. The potential for applying AR to the health care system is enormous, ranging from practice to training. In medical practice, a real-time image of the patient from a scanning system such as MRI or CT scan can be overlaid on the patient's body, making diagnosis more accurate. In the operation room, vital images and information to support the surgeons are available in front or on top of the patient, allowing the surgeon to access that information without having to look away from the patient. For training, there are many areas in healthcare to which AR can be applied for better performance. Examples of AR in medical

\footnotetext{
${ }^{*}$ Corresponding author's email: poonpong@gmail.com, Tel. +66-75-672206, Fax. +66-75-672205 Permalink/DOI: https://dx.doi.org/10.14716/ijtech.v10i7.3267
} 
training range from teaching medical or healthcare students to learn human anatomy using 3D visualization, to helping train healthcare students to master the techniques for checking vital signs. The advantage of using AR in training is that it can be more systematic and specific. Besides that, since marker-based AR can be programmed so that each marker can interact with others, giving the results in terms of data such as distance and time, a trainee can see all the details of the instruction, including their performance, in real time, enabling them to adjust their pace for better performance.

As mentioned, the potential uses of AR for training are numerous and largely untapped; one of them is CPR training. CPR, or Cardiopulmonary Resuscitation, is a lifesaving technique useful for the case in which someone's heartbeat or breathing has stopped due to heart attack. In the United States, more than 350,000 people experience heart attacks each year, and without proper CPR, nine out of ten die. The American Heart Association recommends CPR with chest compressions in the event of witnessing such an incident. For the untrained person, hands-only CPR is enough, entailing 100 to 120 uninterrupted chest compressions a minute. The CPR process keeps oxygenated blood flowing to the brain and other vital organs until more medical treatment can restore normal heart rhythm. There are many ways of receiving training in CPR besides taking a class with a CPR instructor, though it is the best option if time permits. In the case where many people are taking the course or would like to self-train, a CPR training manikin is the best option. The drawback for this option is the cost of the manikin and the restrictions on locations for training. As the number of heart attack victims is very high and the chance of survival is low without proper CPR treatment, we need new and engaging learning methods for CPR education, especially in schools or universities, to increase the number of people who know how to perform proper CPR. Now, there is another option, i.e., using application software, such as popular AR technology. Since there is still some limitation on using the software, which requires some extra equipment such as Microsoft's Hololens or Google glasses, and also due to the reality of the CPR process and environment, in this research we focus on developing an AR system that represents the realistic CPR training environment using the nearly ubiquitous technology of a smartphone. Since CPR is known to be both hard to teach and, once learned, hard to retain, using a smartphone with AR applications makes it possible for people to train any time they want and as many times as they need.

\section{BACKGROUND AND RELATED WORK}

There are two main categories of Augmented Reality (AR): markerless AR, and marker-based AR. By definition, markerless AR is technology that does not need any specific point beforehand for locating the virtual object. The location for placing the virtual object is from the real-world environment, such as the coordinates from GPS devices or the sensor in visual devices. In contrast, marker-based AR is technology that requires a pre-assigned location embedded into the physical object, called a marker, for locating the position of the virtual object. The marker can be in the form of any physical object, as long as it has enough unique visual points. Each category of AR serves different purposes, so the decision of which type of AR to use must be made beforehand, since it may require different types of user interaction. In the case of CPR training, marker-based AR is a suitable choice because the output from the interaction between markers needs accurate data. Research on the applications of interactive AR markers has been done in many areas ranging from education, manufacturing, and medicine. Park et al. (2014) have studied approaches to AR-based user interaction in which rectangular markers were used to support real AR interaction based on fingertip touch using small-sized markers. In this case, the interaction between markers placed on the finger and another on a paper box, which is supposed to be the virtual digital device, has been investigated. The purpose of this study is to find a way to evaluate the design of a digital handheld product without having to make the real prototype. The result 
from this research is accurate and tangible enough to make the touching virtual prototype with fingertip feel realistic. Rahmat et al. (2018) developed an AR application that aims to help children learn the Hijaiyah alphabet using a smartphone and marker, and the result is quite satisfactory. The children found the application appealing. This type of research paves the way for many applications in various fields, medicine being one of them (Madison, 2018; Innovatemedtec, 2018). In the field of medicine, many processes require professional training, and AR can replace traditional practices to save time and yield better performance. Cave et al. (2011) mentioned the importance of CPR training and recommend that secondary schools should have CPR training in the curriculum. Park et al. (2013) propose a new CPR training simulation system that combines both hardware-based sensing and AR-based interactive visualization techniques. With this technique, the trainee can get real-time visual feedback from CPR. The disadvantage of this research is that it requires highly sophisticated hardware such as the CPR manikin, hardware sensor, projectors, and RGB camera, so it is not practical for training many trainees at the same time and not easy to move around. Djajadiningrat et al. (2016) proposed the low-cost "Virtual Trainer," using interactive cloth printed with a life-sized woman manikin for practice and AR as the training instruction guidance on TV. Inside the cloth, there is a pressure sensor and a metronome for speed and pressure guidance during resuscitation. If the speed or pressure is not correct, then voice instruction, such as "press harder," will activate. Even though the manikin cloth can give tangible qualities, it is flat and therefore lacks a realistic feeling. Another disadvantage of this research is that the trainee has to look at the AR display on TV while doing the CPR, which is not practical since he or she has to move their eyes from the virtual victim. Another method for CPR training proposed by Johnson et al. (2018) was the Mix Reality (MR) technique using Microsoft HoloLens. This device has real-time object tracking features and supports holographic superimposition on the real environment, meaning that CPR training on top of the resuscitation manikin with instruction on compression and correct positioning of the hands is possible. The drawback for this set-up is the high cost of HoloLens and the manikin. Recently, the American Heart Association (AHA) and Google have developed a mobile application called My Cardiac Coach ${ }^{\mathrm{TM}}$ for Hands-Only CPR training (Industry Safety and Hygiene News, 2018). With this application, the user can practice Hands-Only CPR at any time, in any place, with their mobile device. The limitation with this tool is that it is available for compatible Android mobile devices only and needs some gaming experience to get good performance. To overcome this burden, we investigated the possibility of developing the CPR training system for everyone, using any smartphone with marker-based AR application.

\section{METHODS}

Although bystander CPR can more than double survival from cardiac arrest, in practice, the prevalence of bystander CPR remains low (Sayre et al., 2008). In traditional CPR, the process consists of the use of chest compressions and mouth-to-mouth breaths. The reluctance to perform CPR by the bystander often includes concerns about disease transmission related to performing mouth-to-mouth ventilation. Recent studies have shown that hands-only CPR is just as effective as the old ABC version of CPR (Hellerman, 2010). In this research, we emphasized hands-only CPR since it is more attractive to bystanders who are usually less willing to give mouth-to-mouth. According to the 2016 "Hands-Only CPR Fact Sheet from the American Heart Association, hands-only CPR has just two easy steps: first, call the emergency number and second, push down hard (five or six centimeters) and fast in the center of the chest at a rate of 100 to 120 compressions per minute. For CPR training using AR to look more realistic and workable, five things need to be considered, including: 
1. Realistic look of the patient

2. The position of the hand on the chest

3. Chest compressions / Number of compressions/minute

4. Pressure on the chest

5. Status display and recording system

The design of the conceptual framework of the system was established and is shown in Figure 1.

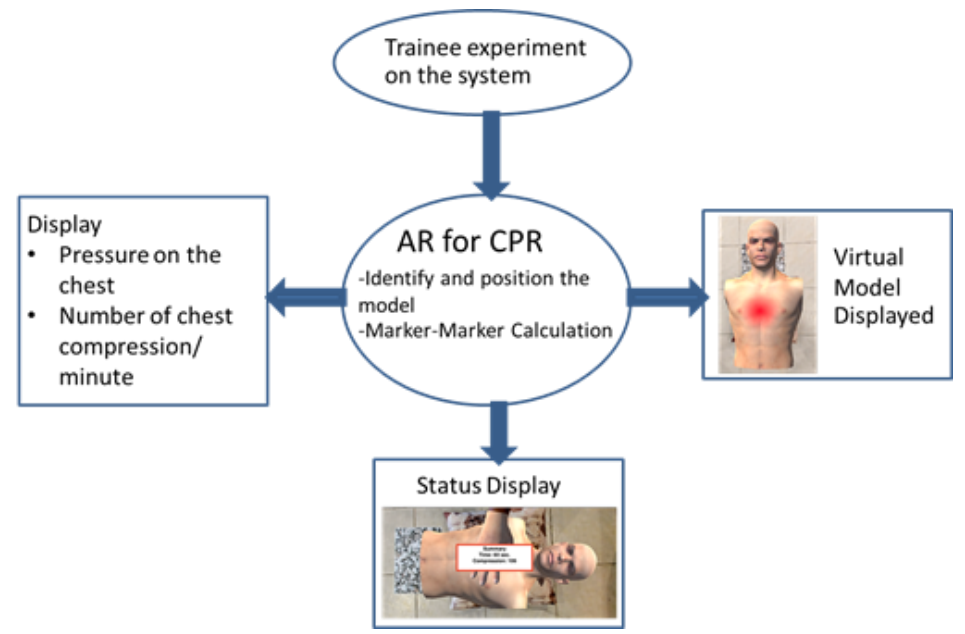

Figure 1 Conceptual framework of the CPR training system using AR technology

\section{EXPERIMENTAL SETUP}

\subsection{System Requirement}

The experimental setup for the marker-based interactive AR system for CPR Training requires both hardware and software to justify the requirement from the conceptual framework mentioned above. In terms of devices aside from the personal computer, iOS, or Android smartphone or tablet are required. For the software, we use Unity 3D cross-platform game engine and Vuforia, which is an augmented reality software development kit (SDK) for mobile devices that enables the creation of augmented reality applications.

To perform the hands-only CPR training using AR technology, we use a pillow and two markers. The pillow represents the patient, so the stiffness of the pad should not be much different from the human body. For the AR markers, the first one represents the virtual model of the human body or the manikin. This marker also represented the coordinate of the floor, i.e., $\mathrm{x}, \mathrm{y}, \mathrm{z}$-axis. The other marker served as the position or the coordinate of the hand, so the distance from the hand and the floor can be calculated (as seen in Figure 2).

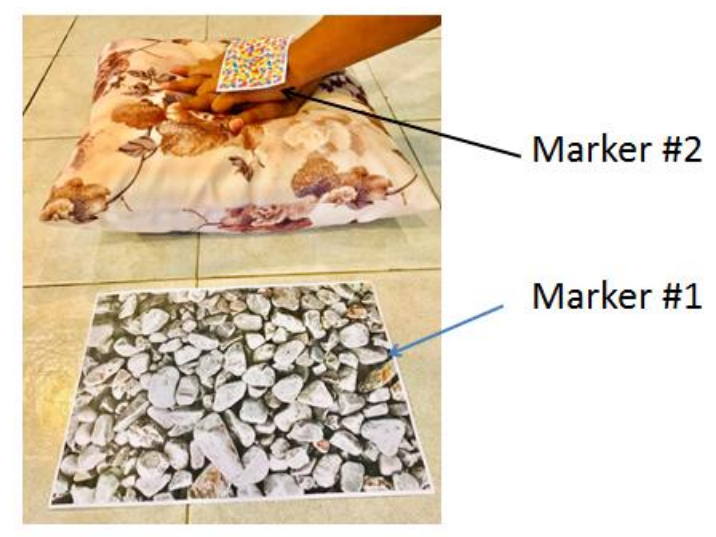

Figure 2 The position of the two AR markers 
The primary function of marker-based augmented reality is the ability to put the virtual model exactly where the position is assigned - in this case, overlaid on top of the pillow. The display output from the AR process is the model of the body of the manikin, which is required in CPR training and looks more realistic than the pillow, as seen in Figure 3.
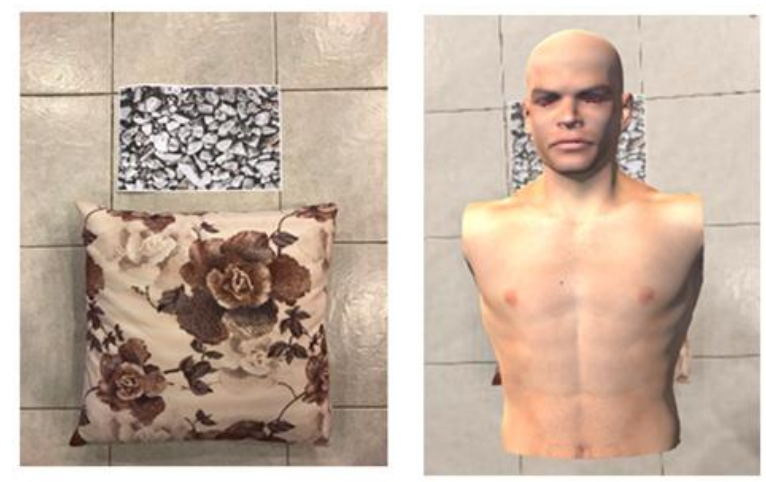

Figure 3 The visual display of the setup before and after AR process

\subsection{Position of the Hand on the Chest}

To make CPR training effective, the position of the hand must be precisely at the end of the persons' breastbone, where the ribs come together. Ordinary people might be afraid to press in the wrong position, so, with augmented reality, the position can be displayed and noticed easily, doing less damage to other parts of the body when doing the CPR process. The presentation for the location of the hands on the chest for the CPR process can be seen when starting the training, as shown in Figure 4.
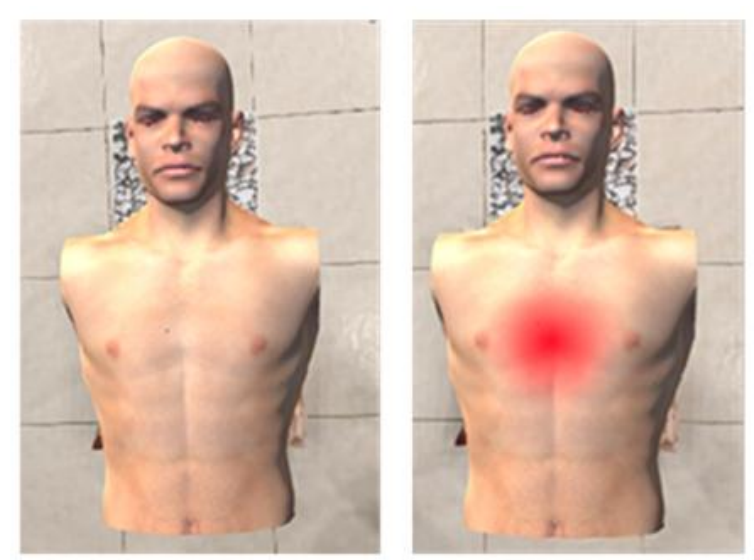

Figure 4 The position needed to be pressed in the CPR process, as seen by the trainee

\subsection{Pressure on the Chest}

As mentioned before, to have an effective result, the depth from the press on the chest should be around five or six centimeters, which is quite difficult for the beginner to guess. With augmented reality technique, the distance is calculated from the two markers; one represents the floor level and the other the level of the hand pressing on the chest (see Figure 5). Because the position of each marker on the $\mathrm{x}, \mathrm{y}$ and $\mathrm{z}$-axes is detected by AR technology, the difference in the $\mathrm{y}$-axis between the two is known.

As seen in Figure 5, the position on the y-axis of the marker \#2 (on the hand) and marker \#1 (on the floor) can be subtracted, giving the depth that has been pressed. When we put the compression on the chest (pillow) the distance between those two markers is closer, so when it reaches five centimeters, the indicator (shown in green circle) will indicate that one cycle of compression is completed, as seen in Figure 6. 

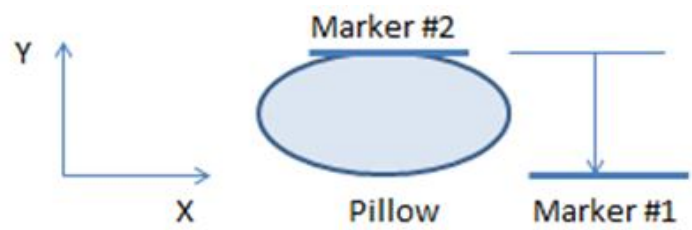

a)

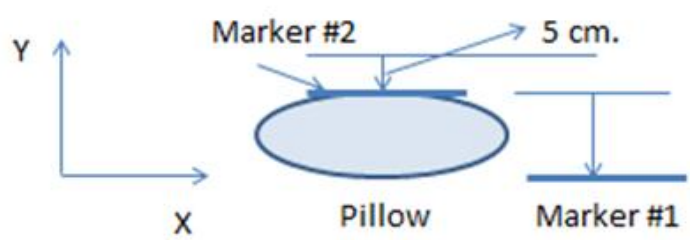

b)

Figure 5 Distance that the trainee's press can be calculated from position of the marker

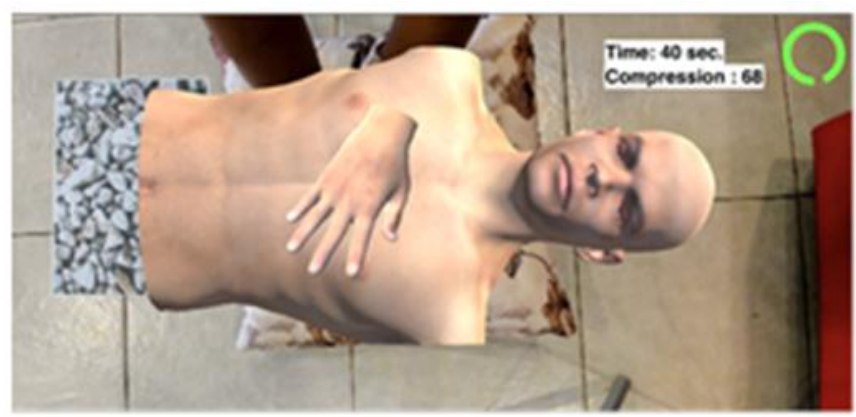

Figure 6 The circle indicator is shown on the top right when finished one cycle of pressing

\subsection{Number of Chest Compressions/Minute}

Since a rate of suitable compression is around 100 to 120 compressions per minute, there should be some indicator to tell the trainee that their activity is too slow, too fast, or not in a periodic pattern to have an effect on the patient. To address this problem, when one compression is completed, the program starts counting and compares to the time of one minute, so the trainee can figure out how to compensate the time and make the regular push to make the 100 compressions per minute goal. The result is shown in Figure 7.

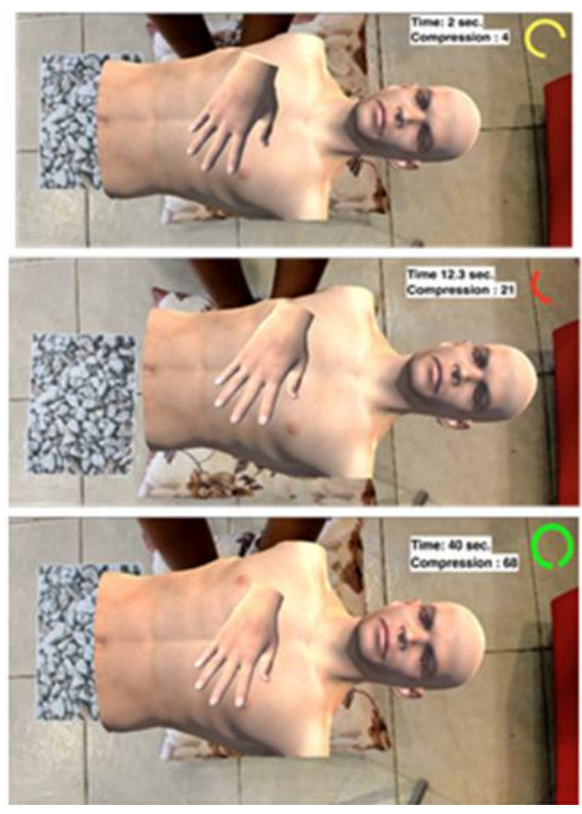

Figure 7 Indication of total rate and time spent 
The indication shown in Figure 7 is the rate of compression in terms of time spent, and numbers of the completed compressions. The picture also illustrates how far the compression goes in one cycle (yellow means start, red means the time is up, and green is complete).

\subsection{Status Display}

After completing one minute of training, the trainee can look at the record to see whether he or she can accomplish the requirement or not. If the rate is not satisfied, he or she can repeat the process. The status display can be adjusted to match the training time, i.e., one minute, five minutes. Figure 8 shows the status for one minute, where we can see that the trainer can accomplish CPR 106 times in one minute.

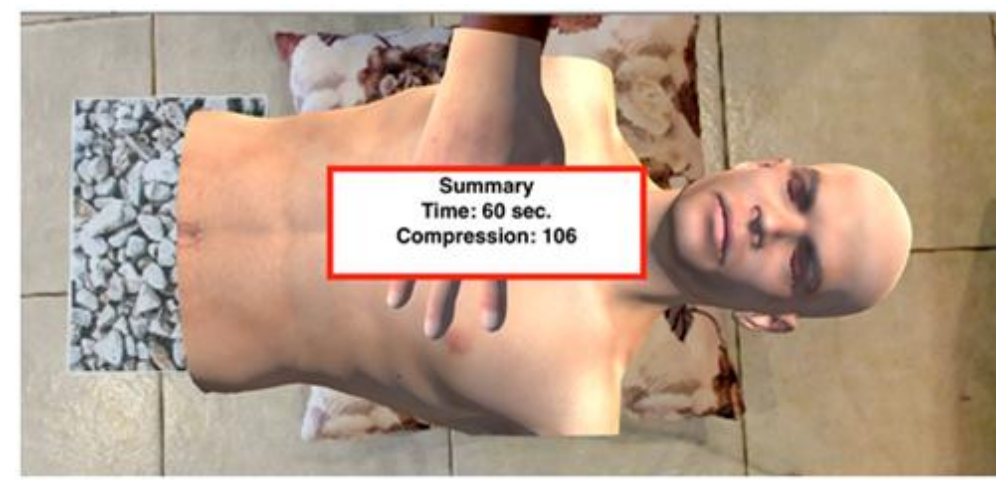

Figure 8 Status display for compression in a period of time

\section{RESULTS AND DISCUSSION}

In order to make sure our CPR training set-up is practical and useful for trainees, we will summarize all the specifications of the system related to the five categories mentioned before. The specifications include the realistic look of the patient, the position of the hand on the chest, the chest compressions and number of compressions/minute, the pressure on the chest, and the status display and recording system.

The realistic look of the virtual patient: This feature is essential for the trainees. If they feel like they are performing CPR on a person, then that person should look and feel like a real person, not a 2D manikin on a flat sheet mat. In our application, there are varieties of the 3D manikin, i.e., male, female, baby, boy, or girl, from which the trainees can choose. For a realistic feeling when performing CPR, trainees can also choose a pillow with a range of stiffness that fits the manikin.

The position of the hand on the chest: This feature is also essential. Most people who do not have experience in CPR training think that they must perform CPR on the chest, closest to the heart, which is incorrect. The right spot is at the end of the person's breastbone, where the ribs come together, which is not easy for everyone to locate. In this application, the right spot is highlighted in red so that the trainees can recognize it easily. After a few trials, the trainees can identify the right spot by themselves. Also, it is necessary to put the hand on that spot for compression. The display for positioning both hands to give chest compressions, such as how to stack the hands or how to lace the fingers of both hands together for proper compression, is also available in AR mode.

The chest compressions and number of compressions/minute: For proper hands-only CPR, the rate of suitable compression is around 100 to 120 compressions per minute. It is not easy for the trainees to keep counting while performing CPR. With this application, the trainees can set the pace that they want to achieve. They can start from a small number and keep going until achieving the goal. 
The pressure on the chest: The technique that we used for calibrating the depth of the press is very accurate within 5\%. The trainees can also vary the depth (or pressure) that they want to perform. They can also change the unit from centimeter to inch as well.

The status display and recording system: The system used AR Technology to support CPR training. All the information about the performance of the training such as the progress of CPR training for each trainee, date and time of the training, and general data (i.e., name, gender, and age) were kept in the database, which can be retrieved for analysis in the future.

The set-up for CPR training using AR technology and the pillow was demonstrated and tested with a group of ten students who had never been trained for CPR before. The students had to wear the AR goggles with iOS or Android smartphones inside for visualization. At first, the average results of the rate, rhythm, and pressure for the CPR process were not right, but after a few trials, the majority of them got it right. Results from the interview stated that the system is easy to use, the instruction and display are easy to follow, and it looks like training with a real person. This type of set-up for CPR training is suitable for self-training. It is also practical for training many people at the same time. The drawback for this training is the bulky goggles that the trainee has to wear. In the future, with the advances in microelectronics and computer technology, practical AR glasses may be available, and any kind of training will be available for everyone.

\section{CONCLUSION}

In this paper, we presented an interactive marker-based AR system for CPR training, which is a low-cost and high-efficiency system for group training. Even though our system used eye goggles connected to iOS or Android mobile phones, which are cumbersome, it is practical enough for users since many people are already using them for playing VR and AR games. Soon, low-cost, lightweight AR glasses with good viewing experience will be available, so CPR training using AR techniques will be among the top choices for users.

Comparing this CPR training using AR technology with other methods (either using CPR training manikins or taking a class with a CPR instructor), it was evident that our approach has some advantages. One example is the accuracy of training in which trainees get all the information about their practice in real time, so they can adjust their performance to meet the goal quickly. Another advantage is cost saving, because the system needs only a mobile phone that almost everybody has, so no need for expensive equipment. In summary, training many people to perform CPR correctly is possible with AR technology, so in the future, with this kind of CPR training application, many lives can be saved.

\section{REFERENCES}

American Heart Association, 2016. Hands-only CPR Fact Sheet. Dallas, Texas, United States Cave, D.M., Aufderheide, T.P., Beeson, J., Ellison, A., Gregory, A., Hazinski, M.F., Hiratzka, L. F., Lurie, K.G., Morrison, L.J., Mosesso, Jr., V.N., Nadkarni, V., Potts, J., Ricardo, A.S., Sayre, M.R., and Schexnayder, S.M., 2013. Importance and Implementation of Training in Cardiopulmonary Resuscitation and Automated External Defibrillation in Schools. Circulation/AHA Journal, Volume 123(6), pp. 691-706

Djajadiningrat, T., Chao, P.Y., Richard, C., Lui, P., 2016. Virtual Trainer: A Low Cost AR Simulation of a Sudden Cardiac Arrest Emergency. In: Proceedings of the 2016 ACM Conference on Designing Interactive Systems. Brisbane, QLD, Australia. ACM, pp. 607618

Hellerman, C., 2010. Hands-only CPR as Effective as Traditional, Studies Show. Available Online at URL: http://www.cnn.com/2010/HEALTH/07/28/chest.compressions/ 
Industry Safety and Hygiene News, 2018. Augmented Reality's CPR Training Unveiled. Available Online at URL: https://www.ishn.com/ articles/108301-Augmented-reality-CPRtraining-unveiled

Innovatemedtec, 2018. Virtual \& Augmented Reality. Available Online at https://innovatemedtec.com/digital-health/virtual-augmented-reality

Johnson, J.G., Rodrigues, D.G., Gubbala, M., Weibel, N., 2018. HoloCPR: Designing and Evaluating a Mixed Reality Interface for Time-critical Emergencies. In: Proceedings of the $12^{\text {th }}$ EAI International Conference on Pervasive Computing Technologies for Healthcare, ACM, pp. 67-76

Madison, D., 2018. The Future of Augmented Reality in Healthcare: Smart Technologies Shaping the Future. Health Management, Volume 18(1), pp. 42-45

Park, H., Jung, H., Park, S., 2014. Tangible AR Interaction based on Fingertip Touch using Smallsized Nonsquare Markers. Journal of Computational Design and Engineering, Volume 1(4), pp. 289-297

Park, N., Kwon, Y., Lee, S., Woo, W., Jeong, J., 2013. Projected AR-Based Interactive CPR Simulator. R. Shumaker (Ed.): VAMR/HCII, Part II, LNCS 8022, pp. 83-89

Rahmat, R.F., Akbar, F., Syahputra, M.F., Budiman, M.A., Hizriadi, A., 2018. An Interactive Augmented Reality Implementation of Hijaiyah Alphabet for Children Education. Journal of Physics: Conference Series, Volume 978 (conference 1), pp. 1-7

Sayre, M.R., Berg, R.A., Cave, D.M., Page, R.L., Potts, J., White, R.D., 2008. Hands-only (Compression-only) Cardiopulmonary Resuscitation: A Call to Action for Bystander Response to Adults Who Experience Out-of-hospital Sudden Cardiac Arrest. Circulation, AHA Journal, Volume 117(16), pp. 2162-2167 